\title{
Aprendizaje basado en Proyectos en Educación Física en Primaria, un estudio de revisión
}

Irene Moya-Mata. Universitat de València
José Peirats Chacón. Universitat de València

Irene Moya-Mata. Universitat de València
José Peirats Chacón. Universitat de València

Recepción: 27/09/2019 | Aceptado: 25/10/2019

Correspondencia a través de ORCID: Irene Moya-Mata

Citar: Moya-Mata, I y Peirats, J (2019). Aprendizaje basado en Proyectos en Educación Física en Primaria, un estudio de revisión. REIDOCREA, 8(2), 115-130.
0000-0002-9428-5179

0000-0002-6580-2712

Resumen: La inclusión de las competencias en el sistema educativo español demanda la aplicación de nuevas metodologías de aprendizaje, centradas en el alumnado; siendo las metodologías activas y concretamente el Aprendizaje basado en Proyectos (ABP), una oportunidad única para responder a estos nuevos retos. El objetivo de este trabajo consiste en revisar, recopilar y analizar la bibliografía relacionada con el ABP en el área de Educación Física (EF), en la etapa de Educación Primaria (EP). Se realizó una revisión documental en las siguientes bases de datos: Dialnet, ERIC, Google Académico, Redalyc, SciElo, Scopus, Sport Discus y Web of Science, seleccionando los documentos publicados entre 2010 y 2019 . La muestra final fue de 18 estudios. Los resultados mostraron que principalmente son experiencias, divulgadas en artículos científicos, y llevadas a cabo en centros públicos de educación infantil y primaria españoles, siendo Aragón la comunidad autónoma con más experiencias publicadas. El tipo de intervención más utilizado es un proyecto anual, abordado a partir del bloque de "juegos y deportes", con la interdisciplinariedad del área de artística, y la competencia digital. En base a los resultados, se puede afirmar que los docentes son cada vez más conscientes del potencial del ABP como herramienta educativa.

Palabras clave: Aprendizaje por proyectos | Metodologías

\section{Project-based learning in Physical Education in Primary, a review study}

\begin{abstract}
The inclusion of competencies in the Spanish education system demands the application of new learning methodologies, centred on students; the actives methodologies being and specifically Projectbased Learning (PBL), a unique opportunity to respond to these new challenges. The objective of this work is to review, compile and analyse the literature related to ABP in the area of Physical Education (PhE), in the Primary Education (PE) stage. A documentary review was carried out in the following databases: Dialnet, ERIC, Google Scholar, Redalyc, SciElo, Scopus, Sport Discus and Web of Science, selecting the documents published between 2010 and 2019. The final sample was 18 studies. The results that are mainly are experiences, disseminated in scientific articles, and carried out in public schools of Spanish primary and primary education, Aragon being the autonomous community with the most published experiences. The most commonly used type of intervention is an annual project, related to the block of "games and sports", with the interdisciplinarity of the artistic area and the digital competence. Based on the results, you can say that teachers are increasingly detected of the potential of PBL as an educational tool.
\end{abstract}

Keywords: Project-based Learning | Active methodologies

\section{Introducción}

El nuevo paradigma educativo vigente basado en las competencias (LOE, 2006; LOMCE, 2013) establece el método por proyectos como una fórmula muy adecuada para desarrollarlas; suponiendo una búsqueda de nuevos modos de hacer, aprender y enseñar para dar respuesta a las necesidades del alumnado. Por ello, las competencias clave dejan de ser un mero concepto en la LOMCE (2013), y se convierten en el punto de inflexión del saber, al saber hacer y del aprender, al aprender a aprender (Trujillo, 2014).

En este nuevo escenario se introducen las metodologías activas, con la finalidad de responsabilizar al alumnado de su proceso de enseñanza, adoptando un papel protagonista frente al método tradicional, al tener que buscar soluciones realistas a 
través de estrategias de búsqueda de información, toma de decisiones y aplicación de los conocimientos adquiridos previamente (De Miguel, 2009).

En estas situaciones, el docente se convierte en un mediador pedagógico entre el alumnado y los conocimientos a adquirir (Julián, Ibor, Aibar y Aguareles, 2017), situando al alumnado en el centro del proceso de aprendizaje, aumentando su participación e implicación, al elegir contenidos y tareas relacionadas con el área, opinar sobre el proceso instructivo o verbalizar las necesidades de aprendizaje, entre otras (Núñez y León, 2015). Y el enfoque se vuelve todavía más valioso en la sociedad actual, al trabajar con alumnado que presenta diferentes niveles de habilidad y diversas características étnicas y/o culturales.

Entre las diferentes metodologías activas que aparecen en este nuevo contexto educativo se encuentra, entre otras, el Aprendizaje Basado en Proyectos o ABP, definida por Trujillo (2015) como una metodología que permite al alumnado adquirir los conocimientos y las competencias clave en el siglo XXI mediante la elaboración de proyectos que dan respuesta a problemas de la vida real.

Las ventajas que presenta esta metodología, según Hernández (2000), son: la construcción de la propia identidad, el desarrollo de la creatividad, el desarrollo del pensamiento crítico, la resolución de problemas o la toma de decisiones entre otras. Por tanto, se puede asegurar que el ABP es un proceso activo y coordinado, que posee la flexibilidad suficiente para adaptarse a las características del alumnado y al contexto sociocultural para resolver un conflicto.

Según García-Varcálcel y Basilotta (2017), es una forma diferente de trabajo que fomenta la indagación, el aprendizaje individual y autónomo del alumnado; ya que adquiere un mayor compromiso por el aprendizaje. Para Blázquez (2016) el trabajo por proyectos fomenta la comunicación y la cooperación entre los agentes que intervienen, y normalmente se realizan en grupos cooperativos, por lo que esta metodología adquiere especial relevancia.

El ABP se puede poner en funcionamiento en cualquier etapa del sistema educativo, desde Educación Infantil hasta la Universidad. Pero es la Primaria, una etapa muy recomendable para iniciarse en este tipo de metodologías, ya que como expone De León (2017), en su estudio sobre la valoración del profesorado de infantil y primaria acerca de esta metodología, el beneficio principal es la gran motivación que genera en el alumnado, además de la versatilidad del método para conectar los aprendizajes del aula con la vida real.

La eficacia y la eficiencia del ABP ha quedado constatada en la etapa de Primaria y en diversas áreas (García Carcedo, 2018; García-Valcárcel y Basilotta, 2017; Murillo, Riaño y Berbel, 2018; Sempere, Rovira-Collado y Baile, 2018;); entendiéndose como un método didáctico integral, holístico y globalizador del aprendizaje (Contreras, 2017). Y la EF puede ser clave para articular estas iniciativas motivantes para implicar al alumnado (Julián et al., 2017); ya que a partir de prácticas motrices se aglutinan diferentes acciones educativas interdisciplinarias, con aplicación en la vida real del alumnado, más allá del patio o el gimnasio.

Y es que las últimas tendencias pedagógicas apuestan por potenciar nuevos enfoques centrados en el aprendizaje constructivista, activo, social y conectado con la vida del alumnado (Fernández-Río, Calderón, Hortigüela, Pérez-Pueyo y Aznar, 2016); siendo el ABP una de las metodologías más idóneas para trabajarse en el área de EF; no sólo por el gran potencial para movilizar aprendizajes escolares, sino además por partir de los intereses y necesidades del alumnado. 
La EF es un área privilegiada al tener a la vida como su referente principal. Y como afirma Julián et al. (2017), las diferentes acciones motrices que se trabajan en esta área, dentro de un estilo de vida activo, culto y saludable en el alumnado constituyen una situación social de referencia, en casi cualquier contexto educativo mediante los proyectos interdisciplinares, a través de actividades físicas, deportivas y artísticoexpresivas.

En el área de EF, los estudios son escasos y bastante recientes, siendo la etapa de Primaria donde más se concentran estos estudios (León-Díaz, Martínez-Muñoz y Santos-Pastos, 2018).

Teniendo en cuenta estas premisas, el objetivo de este estudio consiste en analizar la producción científica publicada en los últimos 10 años (2010-2019) sobre ABP en el área de EF en la etapa de Primaria.

\section{Método}

\section{Diseño}

El diseño utilizado para este estudio ha sido la revisión documental descriptiva (Hernández, Fernández y Baptista, 2010), de los documentos seleccionados.

\section{Procedimiento}

Para realizar la búsqueda bibliográfica se han utilizado diferentes bases de datos de reconocido prestigio en el campo de las Ciencias Sociales como son: Dialnet, ERIC, Google Académico, Redalyc, Scielo, Scopus y Wos.

Debido a la variabilidad terminológica de la metodología ABP se utilizaron los diferentes descriptores encontrados en los estudios encontrados: "aprendizaje basado en proyectos", "proyectos interdisciplinares" y "metodología por proyectos"; además de los descriptores de "educación física" y "primaria".

Para restringir la búsqueda en las bases de datos seleccionadas se introdujo la frase de búsqueda en el título, resumen o palabras claves del artículo. Además, se revisó la lista de referencias bibliográficas de los estudios seleccionados y se revisaron los resúmenes de los estudios obtenidos en las búsquedas.

Los criterios de inclusión utilizados para la selección de los documentos de esta revisión bibliográfica han sido los siguientes: artículos científicos y libros, relacionados con el ABP, contextualizados en el sistema educativo español, concretamente en la etapa de EP y el área de EF, y publicados en los últimos 10 años (2010-2019), con el fin de obtener la información más actual. En cuanto a los criterios de exclusión se eliminaron de la búsqueda los trabajos de fin de grado o máster, comunicaciones a congresos y tesis doctorales, además de estudios publicados antes del 2010, incompletos o repetidos en las diferentes bases de datos.

Todo el proceso fue realizado por una investigadora y supervisado por otro investigador que se encargó de repasar el método de búsqueda, así como de revisar el material excluido de la muestra, corroborando que los criterios aplicados eran coherentes con las finalidades de la investigación. 


\section{Resultados}

De los 1110 documentos encontrados inicialmente, 1092 fueron excluidos por no cumplir con los criterios definidos para este estudio. De esta manera, para el periodo mencionado anteriormente, el análisis se ha centrado en 18 trabajos sobre el ABP en el área de EF y en la etapa de Primaria, publicados en ocho bases de datos científicas diferentes, tal y como se refleja en la siguiente Tabla:

Tabla 1. Distribución según los términos utilizados y las bases de datos empleadas

\begin{tabular}{|c|c|c|c|c|c|c|}
\hline \multirow{2}{*}{$\begin{array}{c}\text { TÉRMINOS } \\
\text { DE } \\
\text { BÚSQUED } \\
\text { A }\end{array}$} & \multicolumn{2}{|c|}{$\begin{array}{c}\text { EDUCACIÓN FÍSICA + } \\
\text { APRENDIZAJE BASADO EN } \\
\text { PROYECTOS + PRIMARIA }\end{array}$} & \multicolumn{2}{|c|}{$\begin{array}{l}\text { EDUCACIÓN FÍSICA + } \\
\text { PROYECTOS } \\
\text { INTERDISCIPLINARES } \\
\text { + PRIMARIA }\end{array}$} & \multicolumn{2}{|c|}{$\begin{array}{c}\text { EDUCACIÓN FÍSICA + } \\
\text { METODOLOGÍA POR } \\
\text { PROYECTOS + PRIMARIA }\end{array}$} \\
\hline & $\begin{array}{l}\mathrm{N}^{\circ} \text { de } \\
\text { resultado } \\
\mathbf{S}\end{array}$ & $\begin{array}{c}\text { Textos } \\
\text { seleccionado } \\
\text { s } \\
\end{array}$ & $\begin{array}{l}\mathbf{N}^{\circ} \text { de } \\
\text { resultado } \\
\mathbf{S}\end{array}$ & $\begin{array}{c}\text { Textos } \\
\text { seleccionado } \\
\mathbf{S}\end{array}$ & $\begin{array}{l}N^{\circ} \text { de } \\
\text { resultado } \\
\mathbf{S}\end{array}$ & $\begin{array}{c}\text { Textos } \\
\text { seleccionado } \\
\mathbf{s} \\
\end{array}$ \\
\hline Dialnet & 28 & 5 & 6 & 1 & 4 & 2 \\
\hline ERIC & 0 & 0 & 0 & 0 & 0 & 0 \\
\hline $\begin{array}{c}\text { Google } \\
\text { académico }\end{array}$ & 486 & 5 & 296 & 2 & 261 & 1 \\
\hline Redalyc & 1 & 0 & 17 & 0 & 1 & 1 \\
\hline Scielo & 1 & 1 & 0 & 0 & 0 & 0 \\
\hline Scopus & 2 & 0 & 0 & 0 & 0 & 0 \\
\hline $\begin{array}{c}\text { Sport } \\
\text { Discus }\end{array}$ & 6 & 0 & 1 & 0 & 0 & 0 \\
\hline Wos & 0 & 0 & 0 & 0 & 0 & 0 \\
\hline TOTAL & 524 & 11 & 320 & 3 & 266 & 4 \\
\hline
\end{tabular}

\section{Evolución de la producción científica}

La revisión realizada nos permite afirmar que en el 2011 comienzan los estudios sobre el ABP, en el área de EF y en la etapa de Primaria (Juez y De los Santos, 2011). Pero hasta el 2017, sólo encontramos entre uno y dos estudios como máximo. En el 2017 encontramos 17 estudios sobre la temática analizada. Y en los años posteriores, hay un ligero aumento al encontrar tres estudios en cada uno de los años analizados: 2018 y hasta septiembre del 2019, que se finaliza la búsqueda.

\section{Tipo de trabajo}

Sólo se ha encontrado un estudio de investigación sobre ABP en EF en la etapa de Primaria, concretamente el de García y Baena-Extremera (2017). Es un estudio cuyo objetivo es conocer en qué medida la motivación del alumnado de EF es mayor o menor, a partir de un trabajo por competencias basado en un proyecto, o a través de una metodología tradicional.

En cambio, se han encontrado cinco estudios con propuestas didácticas para ser llevadas a la práctica, en donde: 1) Álvarez e Yllera (2017) exponen cuáles son los beneficios de la implementación de la performance en el aula de primaria; 2) Carrera (2016) muestra la idea de que el alumnado es capaz de crear nuevos deportes a través 
del trabajo en equipo y la modificación de los elementos estructurales del juego deportivo; 3) Gil y Sáez (2017) proponen una UD sobre los juegos tradicionales de Castilla La Mancha a partir de los juegos que aparecen en la obra del Quijote; 4) González-Víllora y Prieto-Ayuso (2017) diseñan un proyecto para trabajar a través del tenis la interdisciplinariedad; y 5) Rodríguez-Martín y Buscà (2018) aportan una serie de orientaciones didácticas para trabajar interdisciplinariamente la EF y las matemáticas.

El grueso de los estudios analizados se relaciona con experiencias prácticas llevadas a cabo en centros escolares de Primaria. Concretamente son 12 trabajos en donde: 1) Abad, Campo, Cortés y Lienas (2017) realizan un proyecto interdisciplinar basado en el cortometraje; 2) Castellar, Pradas, Rapún, Coll y Pérez (2013) muestran un proyecto educativo articulado al uso de la bicicleta; 3) González, Villar, Pastor y Gil (2013) exponen una propuesta teórica-práctica entre las áreas de EF e Inglés; 4) Juez y De los Santos (2011) desarrollan la práctica la dinamización de textos teatrales y cuentos con el área de EF; 5) Peixoto (2014) presenta un proyecto interdisciplinar a partir de los Juegos Olímpicos de Londres 2012; 6) Peixoto-Pino, Rico-Díaz y Arufe (2019) enseñan un proyecto basado en la prevención de accidentes y los primeros auxilios en la provincia de Pontevedra; 7) Pérez-Herráez y Valencia-Peris (2019) describen una experiencia sobre los juegos tradicionales valencianos; 8) Ramírez, Padial, Torres, Chinchilla y Cepero (2018) presentan el efecto que causó en el desarrollo de la competencia digital una intervención desde el área de EF a través de la metodología ABP; 9) Rodríguez Cabanillas (2017) expone su proyecto interdisciplinar con el senderismo como hilo conductor y la EF su máximo exponente; 10) Rosa-Guillamón, Carrillo-López y García-Cantó (2019) muestran su proyecto sobre las actividades físicas en el medio natural, como muestra del desarrollo integral del alumnado; 11) Sánchez y López (2017) integran la salud tanto individual como colectiva, con el cuidado del planeta Tierra y la gestión medioambiental y sostenibilidad; y 12) Segovia y Gutiérrez Díaz del Campo (2018) comprueban cómo las competencias clave sociales y cívicas, y el sentido de la iniciativa y espíritu emprendedor mejoran tras implementar una UD de Educación Deportiva por Proyectos en un grupo de $2^{\circ}$ curso.

\section{Tipo de centro y localización}

El tipo de centro que predomina en estas intervenciones es principalmente un Centro de Educación Infantil y Primaria (CEIP), con nueve estudios (Castellar et al., 2013; García y Baena-Extremera, 2017; González et al., 2013; Peixoto, 2014; Peixoto-Pino et al., 2019; Pérez-Herráez y Valencia-Peris, 2019; Ramírez et al., 2018; Rosa-Guillamón et al., 2019; Segovia y Gutiérrez Díaz del Campo, 2018); frente a los tres estudios en un Centro Rural Agrupado (CRA) (Abad et al., 2017; Juez y De los Santos, 2011; Rodríguez Cabanillas, 2017), o un estudio en un Centro de Innovación educativa (CRIE) (Sánchez y López, 2017). En cambio, existen cinco estudios que no especifican el tipo de centro (Álvarez e Yllera, 2017; Carrera, 2016; Gil y Sáez, 2017; González-Víllora y PrietoAyuso, 2017; Rodríguez y Buscà, 2018).

Siendo Aragón la Comunidad Autónoma española que más estudios recoge (Abad et al., 2017; Castellar et al., 2013; Rodríguez Cabanillas, 2017); seguida de Castilla La Mancha (González et al., 2013; Segovia y Gutiérrez Díaz del Campo, 2018), Castilla y León (Juez y De los Santos, 2011; Sánchez y López, 2017), Murcia (García y BaenaExtremera, 2017; Rosa-Guillamón et al., 2019), Galicia (Peixoto, 2014; Peixoto-Pino et 
al., 2019); y por último, Andalucía (Ramírez et al., 2018) y Comunidad Valenciana (Pérez-Herráez y Valencia-Peris, 2019).

\section{Nivel educativo}

En seis estudios, las propuestas y/o intervenciones se llevan a cabo en varios cursos. Concretamente, la intervención de Peixoto-Pino et al. (2019) se aplica en la etapa de Infantil y Primaria; Abad et al. (2017) en la etapa de Primaria; Castellar et al. (2013) de $3^{\circ}$ a $6^{\circ}$ curso; González et al. (2013) de $4^{\circ}$ y $5^{\circ}$ curso; y los proyectos de González-Víllora y Prieto-Ayuso (2017), y Sánchez y López (2017) en los cursos de $5^{\circ}$ y $6^{\circ}$ de Primaria.

En cambio, tanto en $6^{\circ}$ curso (Pérez-Herráez y Valencia-Peris, 2019; Ramírez et al., 2018; Segovia y Gutiérrez Díaz del Campo, 2018) como en $4^{\circ}$ curso (Álvarez e Yllera, 2017; Juez y De Los Santos, 2011; Rosa-Guillamón et al., 2019), se llevan a cabo tres proyectos. Y en el $2^{\circ}$ curso sólo encontramos un estudio (García y Baena-Extremera, 2017).

En necesario comentar que nos encontramos con cinco estudios en donde no se concreta el curso al cual se dirige la intervención en Primaria (Carrera, 2016; Gil y Sáez, 2017; Peixoto, 2014; Rodríguez Cabanillas, 2017; Rodríguez-Martín y Buscà, 2018).

\section{Tipo de intervención}

La intervención que más se ha aplicado y propuesto en estos estudios es la de un proyecto llevado a cabo a lo largo de un curso escolar con nueve estudios (Abad et al., 2017; Álvarez e Yllera, 2017; Gil y Sáez, 2017; González-Víllora y Prieto-Ayuso, 2017; Peixoto, 2014; Peixoto-Pino et al., 2019; Pérez-Herráez y Valencia-Peris, 2019; Rodríguez Cabanillas, 2017; Segovia y Gutiérrez Díaz del Campo, 2018).

El siguiente tipo de intervención sería el diseño y aplicación de una UD, con cuatro estudios (Carrera, 2016; González et al., 2013; Juez y De los Santos, 2011; RosaGuillamón et al., 2019).

En menor medida encontramos otro tipo de intervenciones como son:

- Diseño y aplicación de 2 UUDD, en donde a través de la comparativa de dos grupos de $2^{\circ}$ curso de EP, mediante la aplicación de dos UUDD (una al grupo experimental mediante proyectos y otra al grupo control mediante enseñanza tradicional), se comprueba si la motivación del alumnado mejora (García y Baena-Extremera, 2017)

- Un proyecto llevado a cabo durante dos cursos escolares, en donde a partir de la salud se pretende concienciar al alumnado del cuidado del medio ambiente, con el proyecto "SaludArte" (primer año); y de la eficiencia energética y sostenible, con el proyecto "Energía Sostenible: 3.Sol" (segundo año) (Sánchez y López, 2017).

- Un proyecto llevado a cabo durante cuatro cursos escolares, en donde a partir del proyecto "Aula en bici", articulado a partir del contenido de la utilización de la bicicleta, en los cursos de $3^{\circ}$ a $6^{\circ}$ de Primaria, desde una triple perspectiva: como medio de transporte urbano, como modalidad deportiva ecológica y saludable, y como herramienta para descubrir el medio natural (Castellar et al., 2013). 
- Un proyecto sobre la mejora de la competencia digital, a partir de tres micro proyectos, uno por trimestre, denominados: "Circus" (primer trimestre) con acroesport, habilidades gimnásticas, mimo y malabares; "Marbella" (segundo trimestre) con contenidos de baloncesto, voleibol, tenis, ciclismo, patines y senderismo; y finalmente "Juegos Olímpicos" (tercer trimestre) con el trabajo del atletismo, la gimnasia, el tenis de mesa y el fútbol (Ramírez et al., 2018).

- Y por último, encontramos un estudio en donde sólo se dan unas orientaciones didácticas para el trabajo de la competencia matemática en cada bloque de contenidos del área de EF, con ejemplos de actividades tipo, pero sin concretarlo ni temporalizarlo (Rodríguez-Martín y Buscà, 2018).

\section{Competencias clave}

Las competencias a las que hacen referencia los estudios analizados sobre ABP en EF en la etapa de Primaria son, de mayor a menos tratamiento, las siguientes:

- Competencia digital ( $n=5$ ): Gil y Sáez (2017), González et al. (2013), GonzálezVíllora y Prieto-Ayuso (2017), Ramírez et al. (2018) y Rosa-Guillamón et al. (2019).

- Competencias sociales y cívicas ( $n=5)$ : Gil y Sáez (2017), González et al. (2013), González-Víllora y Prieto-Ayuso (2017), Rosa-Guillamón et al. (2019), y Segovia y Gutiérrez Díaz del Campo (2018).

- Comunicación lingüística ( $=$ 4): Gil y Sáez (2017), González et al. (2013), González-Víllora y Prieto-Ayuso (2017) y Rosa-Guillamón et al. (2019).

- Sentido de la iniciativa y espíritu emprendedor ( $n=4)$ : González et al. (2013), González-Víllora y Prieto-Ayuso (2017), Rosa-Guillamón et al. (2019) y Segovia y Gutiérrez Díaz del Campo (2018).

- Conciencia y expresiones culturales ( $n=4)$ : Gil y Sáez (2017), González et al. (2013), González-Víllora y Prieto-Ayuso (2017) y Rosa-Guillamón et al. (2019).

- Competencia matemática y en ciencia y tecnología $(\mathrm{n}=3)$ : González et al. (2013), Rodríguez-Martín y Buscà (2018) y Rosa-Guillamón et al. (2019).

- Aprender a aprender ( $\mathrm{n}=3$ ): González et al. (2013), González-Víllora y PrietoAyuso (2017) y Rosa-Guillamón et al. (2019).

\section{Contenidos de EF}

Los estudios seleccionados utilizan la EF como hilo conductor de sus proyectos. Los contenidos más utilizados en los trabajos de ABP en la etapa de Primaria los hemos agrupado en los siguientes bloques y con sus respectivos porcentajes:

- Los juegos y actividades deportivas $(n=10)$. De los 10 trabajos encontrados, 4 hacen referencia a los juegos; bien a través de orientaciones didácticas para introducir las matemáticas en los juegos de EF (Rodríguez-Martín y Buscà, 2018), o bien desde la tradicionalidad, como es el caso de los juegos tradicionales manchegos (Gil y Sáez, 2017), valencianos (Pérez-Herráez y Valencia-Peris, 2019), o del mundo (Segovia y Gutiérrez Díaz del Campo, 2018). 
En cambio, los seis estudios encontrados relacionados con el bloque de los deportes, los proyectos abordan contenidos como la bicicleta (Castellar et al., 2013), el floorball (González et al., 2013), el olimpismo (Peixoto, 2014), la creación de nuevos deportes (Carrera, 2016), el tenis (González-Víllora y PrietoAyuso, 2017), o un compendio de diversos deportes (Ramírez et al., 2018), a través del proyecto "Juegos Olímpicos", con deportes como el atletismo, la gimnasia, el tenis de mesa y el fútbol; o el proyecto "Marbella" con deportes como el baloncesto, el voleibol, el tenis y el ciclismo.

- La expresión corporal $(n=8)$. Dentro de este bloque encontramos dos proyectos que abordan la dramatización, como es el estudio de Juez y De los Santos (2011) a través de espacios de fantasía y cuentos infantiles; y Rodríguez-Martín y Buscà (2018) a través de la competencia matemática. Otra manifestación artística que aparece en estos estudios es el mimo, el cual es utilizado por Ramírez et al. (2018), a través del proyecto "Circus". Respecto a la expresión corporal es utilizada en los estudios de Abad et al. (2017) a través del cortometraje; y García y Baena-Extremera (2017) mediante una UD aplicada a $2^{\circ}$ de EP para conocer la motivación del alumnado ante una metodología por proyectos o tradicional. Por último, Álvarez e Yllera (2017) trabajan a partir de la performance, como mejora de las habilidades expresivo-comunicativas y la creatividad.

- La salud ( $\mathrm{n}=5)$. De los cinco estudios encontrados, cuatro abordan los contenidos generales relacionados con la salud del alumnado, es decir: la alimentación saludable, higiene personal y postural, y la actividad física saludable (Castellar et al. 2013; García y Baena-Extremera, 2017; RodríguezMartín y Buscà, 2018; Sánchez y López, 2017); aunque en este último estudio integran la salud con la conciencia medioambiental y la sostenibilidad. Y el proyecto de Peixoto-Pino et al. (2019) lo abordan desde la prevención de accidentes y los primeros auxilios.

- Las actividades en el medio natural $(\mathrm{n}=3)$. En este bloque, el senderismo vertebra dos de los tres proyectos encontrados (Ramírez et al., 2018; Rodríguez Cabanillas, 2017); y en el caso de Rosa-Guillamón et al. (2019), parten del centro de interés de las actividades físicas en el medio natural, para desarrollar las competencias clave, a través de diversas actividades como son: el senderismo, el kayak, el piragüismo, el pádel-surf, la orientación, la espeleología y la bicicleta de montaña.

- El conocimiento del cuerpo $(n=2)$. En el proyecto de García y Baena-Extremera (2017) se trabaja con una UD sobre esquema corporal, lateralidad sentidos y equilibrio; mientras que Rodríguez-Martín y Buscà (2018) abordan el conocimiento del cuerpo.

- Por último, Ramírez et al. (2018), abordan otras actividades ( $\mathrm{n}=2$ ), como son los malabares, a través del proyecto "Circus", y los patines a través del proyecto "Marbella".

\section{Interdisciplinariedad}

Los contenidos de EF, a su vez, se interrelacionan con otras áreas del currículo, tal y como justifican la mayoría de los proyectos. Siendo las áreas curriculares, de más a menos trabajadas interdisciplinarmente, las siguientes: 
- Educación Artística $(\mathrm{n}=15)$. Concretamente en Plástica se concentran 10 estudios: Abad et al. (2017), Álvarez e Yllera (2017), Gil y Sáez (2017), González-Víllora y Prieto-Ayuso (2017), Juez y De los Santos (2011), Peixoto (2014), Peixoto-Pino et al. (2019), Pérez-Herráez y Valencia-Peris (2019), Rodríguez Cabanillas (2017), Segovia y Gutiérrez Díaz del Campo (2018); y en Música, cinco estudios: Abad et al. (2017), Álvarez e Yllera (2017), Gil y Sáez (2017), González-Víllora y Prieto-Ayuso (2017) y Peixoto (2014).

- Lengua Castellana y Literatura ( $\mathrm{n}=11$ ): Abad et al. (2017), Álvarez e Yllera (2017), Gil y Sáez (2017), González-Víllora y Prieto-Ayuso (2017), Juez y De los Santos (2011), Peixoto (2014), Peixoto-Pino et al. (2019), Pérez-Herráez y Valencia-Peris (2019), Rodríguez Cabanillas (2017), Rosa-Guillamón et al. (2019), y Segovia y Gutiérrez Díaz del Campo (2018).

- Ciencias de la Naturaleza ( $\mathrm{n}=9$ ): Castellar et al. (2013), Álvarez e Yllera (2017), Gil y Sáez (2017), González-Víllora y Prieto-Ayuso (2017), Peixoto (2014), Peixoto-Pino et al. (2019), Rodríguez Cabanillas (2017), Rosa-Guillamón et al. (2019), y Sánchez y López (2017).

- Matemáticas ( $n=7$ ): Castellar et al. (2013), Abad et al. (2017), Álvarez e Yllera (2017), Gil y Sáez (2017), González-Víllora y Prieto-Ayuso (2017), Juez y De los Santos (2011), Peixoto (2014), Peixoto-Pino et al. (2019), Pérez-Herráez y Valencia-Peris (2019), Rodríguez-Martín y Buscà (2018) y Rosa-Guillamón et al. (2019).

- Ciencias Sociales ( $n=6)$ : Abad et al. (2017), Gil y Sáez (2017), González-Víllora y Prieto-Ayuso (2017), Rodríguez Cabanillas (2017), Rosa-Guillamón et al. (2019), y Segovia y Gutiérrez Díaz del Campo (2018).

- Lengua Extranjera: concretamente es el inglés el idioma abordado en todos los estudios encontrados ( $n=5$ ): González et al. (2013), González-Víllora y PrietoAyuso (2017), Peixoto (2014), Peixoto-Pino et al. (2019) y Rosa-Guillamón et al. (2019).

- Religión y Valores Sociales y Cívicos $(\mathrm{n}=3$ ): Castellar et al. (2013), Peixoto (2014) y Rosa-Guillamón et al. (2019).

\section{Síntesis de resultados alcanzados}

- Sobre la evolución de la producción científica, se puede afirmar que es muy reciente, concentrándose exclusivamente en la actual década.

- En cuanto al tipo de trabajos encontrados, destacan mayoritariamente las experiencias prácticas realizadas en los centros de Primaria; seguido por los estudios realizados sobre propuestas didácticas para ser llevadas a la práctica en los centros escolares y, por último, se señala que sólo se ha encontrado un caso de investigación sobre ABP en Educación Física.

- Si nos fijamos en el apartado de tipo de centro, se subraya que principalmente es el Centro de Educación Infantil y Primaria, seguido a bastante distancia por el Centro Rural Agrupado, y quedando el Centro de Innovación Educativa en último lugar. Su localización, en cuanto a número de casos y por comunidad autónoma, la encabeza Aragón, seguida por Castilla La Mancha, Castilla y León, Murcia, Galicia, Andalucía y la Comunidad Valenciana. 
- En estas propuestas y/o intervenciones predominan aquéllas que se llevan a cabo en varios cursos; seguidas de las aplicadas en $6^{\circ}$ y $4^{\circ}$ curso de Primaria, y por último en $2^{\circ}$ curso. Existiendo, también, estudios que no concretan el nivel educativo.

- Y el tipo de intervención se concreta, mayoritariamente, en el proyecto desarrollado a lo largo de un curso escolar. Seguido por el diseño y aplicación de una Unidad Didáctica y por un conjunto diverso de intervenciones que abarcan mayor número de cursos, comparaciones entre grupos o varios proyectos en el mismo curso escolar.

- Las competencias clave abordadas se reparten casi equitativamente entre la digital, las sociales y cívicas, la lingüística, el sentido de la iniciativa y espíritu emprendedor, la conciencia y expresiones culturales, la competencia matemática y en ciencia y tecnológica, y el aprender a aprender.

- Los contenidos de EF más utilizados, agrupados por bloques y en orden de mayor número de casos han sido los juegos y actividades deportivas, la expresión corporal, la salud, las actividades en el medio natural, y el conocimiento del cuerpo.

- Para finalizar, en cuanto a la interdisciplinariedad, las áreas curriculares más relacionadas son la Educación Artística, Lengua Castellana y Literatura, Ciencias de la Naturaleza, Matemáticas, Ciencias Sociales, Lengua Extranjera y Religión y Valores Sociales y Cívicos.

A continuación, se presenta la Tabla 2, en donde se incluye la información más relevante sobre los 18 trabajos seleccionados. Esta tabla incluye: a) los autores del estudio y el año de publicación; b) objetivo principal del estudio; c) datos sobre la muestra (número de alumnado, curso y tipo de centro); d) el proyecto o intervención realizada (tipo de intervención, título del proyecto y duración); y e) los resultados obtenidos. 
Tabla 2. Información relevante de los estudios seleccionados

\begin{tabular}{|c|c|c|c|c|}
\hline $\begin{array}{l}\text { Autorles } \\
\text { (año) }\end{array}$ & Objetivo principal & Muestra & Proyecto e Intervención & Resultados \\
\hline $\begin{array}{l}\text { Juez y De los } \\
\text { Santos (2011) }\end{array}$ & $\begin{array}{l}\text { Desarrollar el currículo mediante } \\
\text { propuestas interdisciplinares y las } \\
\text { competencias básicas }\end{array}$ & $\begin{array}{l}13 \text { alumnos/as } \\
\left(4^{\circ} \text { curso }\right) \\
\text { CRA }\end{array}$ & $\begin{array}{l}\text { UD con estrategias de trabajo cooperativo } \\
\text { y autónomo, y actividades artístico- } \\
\text { expresivas, a través de los cuentos e } \\
\text { historietas infantiles }\end{array}$ & $\begin{array}{l}\text { La experiencia constata que se pueden educar } \\
\text { las emociones desde este tipo de proyectos, y } \\
\text { fomentar los contenidos expresivos desde el } \\
\text { área de EF }\end{array}$ \\
\hline $\begin{array}{l}\text { González et al. } \\
\text { (2013) }\end{array}$ & $\begin{array}{l}\text { Contrastar el mejor aprendizaje de } \\
\text { la lengua extranjera, bien a través } \\
\text { del trabajo de clase; o bien con } \\
\text { situaciones integradas en EF }\end{array}$ & $\begin{array}{l}49 \text { alumnos/as } \\
4^{\circ} \text { curso }(n=24) \\
5^{\circ} \text { curso }(n=25) \\
\text { CEIP }\end{array}$ & $\begin{array}{l}\text { Unidad Didáctica compuesta por } 5 \\
\text { sesiones de floorball, aplicada en } 4^{\circ} \text { curso } \\
\text { (grupo de innovación docente); y } 5^{\circ} \text { curso } \\
\text { (grupo control) }\end{array}$ & $\begin{array}{l}\text { En el grupo experimental se comprobó una } \\
\text { mayor progresión en la adquisición del inglés y } \\
\text { de la capacidad de atención, comprensión y } \\
\text { ejecución de conceptos prácticos y de trabajo }\end{array}$ \\
\hline $\begin{array}{l}\text { Castellar et al. } \\
(2013)\end{array}$ & $\begin{array}{l}\text { El desarrollo y la aplicación } \\
\text { didáctica de un proyecto } \\
\text { educativo que articule un } \\
\text { contenido vinculado a la bicicleta }\end{array}$ & $\begin{array}{l}3^{\circ}-6^{\circ} \text { curso } \\
12 \text { CEIPs }\end{array}$ & $\begin{array}{l}\text { Proyecto Educativo: "Aula en bici", } \\
\text { centrado en la integración del uso didáctico } \\
\text { de la bicicleta, con los hábitos saludables y } \\
\text { la educación de la conciencia } \\
\text { medioambiental }\end{array}$ & $\begin{array}{l}\text { El enfoque didáctico del uso de la bicicleta: } \\
\text { como medio de transporte, modalidad deportiva } \\
\text { o forma de descubrir el entorno natural y } \\
\text { urbano, facilita una aplicación más global de } \\
\text { este tipo de contenidos. }\end{array}$ \\
\hline Peixoto (2014) & $\begin{array}{l}\text { Transmitir los valores del deporte } \\
\text { y conocer el movimiento olímpico }\end{array}$ & $\begin{array}{l}\text { Diferentes } \\
\text { cursos de } \\
\text { Primaria } \\
\text { CEIP }\end{array}$ & $\begin{array}{l}\text { Proyecto interdisciplinar llevado a cabo en } \\
\text { un colegio, durante el curso escolar } 11 / 12\end{array}$ & $\begin{array}{l}\text { Proyecto muy adecuado para trabajar la } \\
\text { convivencia y el deporte olímpico en toda la } \\
\text { comunidad educativa }\end{array}$ \\
\hline Carrera (2016) & $\begin{array}{l}\text { Crear deportes alternativos } \\
\text { mediante la metodología ABP }\end{array}$ & No se especifica & Creación de un nuevo deporte & $\begin{array}{l}\text { La creación de nuevos deportes es viable } \\
\text { mediante las metodologías activas, el trabajo } \\
\text { en equipo y la modificación de elementos } \\
\text { estructurales del deporte }\end{array}$ \\
\hline Abad et al. (2017) & $\begin{array}{l}\text { Crear, diseñar y llevar a la } \\
\text { práctica un proyecto } \\
\text { interdisciplinar, mediante la } \\
\text { temática del cine, y } \\
\text { concretamente, el cortometraje }\end{array}$ & $\begin{array}{l}7 \text { alumnos } \\
\left(\text { El- }-1^{\circ}\right) \\
8 \text { alumnos } \\
\left(2^{\circ}-6^{\circ}\right) \\
\text { CRA }\end{array}$ & $\begin{array}{l}\text { Proyecto interdisciplinar llevado a cabo } \\
\text { durante un curso escolar sobre el cine }\end{array}$ & $\begin{array}{l}\text { Los proyectos interdisciplinares, además de } \\
\text { abordar aspectos curriculares de las diferentes } \\
\text { áreas, se potencian valores como: capacidad } \\
\text { de esfuerzo y superación, trabajo en equipo, } \\
\text { creatividad, empatía... }\end{array}$ \\
\hline $\begin{array}{l}\text { Álvarez e Yllera } \\
(2017)\end{array}$ & $\begin{array}{l}\text { Mostrar cuáles son los beneficios } \\
\text { de la implementación de la } \\
\text { performance en el aula de } \\
\text { primaria }\end{array}$ & $4^{\circ}$ curso & $\begin{array}{l}\text { Proyecto Educativo: "Animales en acción" } \\
\text { A partir de la performance en diferentes } \\
\text { áreas curriculares }\end{array}$ & $\begin{array}{l}\text { La performance es un recurso pedagógico } \\
\text { altamente motivador y fácilmente } \\
\text { implementable en cualquier área de la etapa de } \\
\text { Educación Primaria }\end{array}$ \\
\hline
\end{tabular}




\begin{tabular}{|c|c|c|c|c|}
\hline $\begin{array}{l}\text { García y Baena- } \\
\text { Extremera (2017) }\end{array}$ & $\begin{array}{l}\text { Conocer en qué medida, la } \\
\text { motivación del alumnado de EF es } \\
\text { mayor o menor, a partir de una } \\
\text { metodología tradicional o un } \\
\text { trabajo por proyecto }\end{array}$ & $\begin{array}{l}37 \text { alumnos/as } \\
\left(2^{\circ} \text { curso }\right)\end{array}$ & $\begin{array}{l}2 \text { UUDD en el tercer trimestre: } \\
\text { En la UD-1 (7s): esquema corporal, } \\
\text { lateralidad, expresión corporal y sentidos. } \\
\text { En la UD-2 (7s): equilibrio y salud }\end{array}$ & $\begin{array}{l}\text { Mejora en la motivación del alumnado al } \\
\text { trabajar por competencias, que de manera } \\
\text { tradicional; encontrando diferencias } \\
\text { significativas en el gusto por las clases y el } \\
\text { aprendizaje de nuevos juegos }\end{array}$ \\
\hline Gil y Sáez (2017) & $\begin{array}{l}\text { Trabajar los contenidos motrices y } \\
\text { etnográficos del siglo XVI, a } \\
\text { través de la obra del Quijote }\end{array}$ & Primaria & $\begin{array}{l}\text { "Los juegos populares y tradicionales de } \\
\text { Don Quijote de la Mancha" } \\
\text { (Proyecto anual: } 53 \text { sesiones) }\end{array}$ & $\begin{array}{l}\text { Con esta propuesta se pretende hacer al } \\
\text { alumnado partícipe del proceso de búsqueda, } \\
\text { diseño y adaptación de los juegos } \\
\text { referenciados en el Quijote }\end{array}$ \\
\hline $\begin{array}{l}\text { González-Víllora y } \\
\text { Prieto-Ayuso } \\
\text { (2017) }\end{array}$ & $\begin{array}{l}\text { Mejorar los contenidos técnico- } \\
\text { tácticos de los deportes de } \\
\text { raqueta, y las habilidades sociales } \\
\text { del alumnado }\end{array}$ & $5^{\circ}$ y $6^{\circ}$ Primaria & $\begin{array}{l}\text { "Desarrolla tu propio equipo de Copa } \\
\text { Davis": } \\
\text { Pretemporada: } 4 \text { sesiones, Temporada: } 8 \\
\text { sesiones y Fase Final: } 4 \text { sesiones }\end{array}$ & $\begin{array}{l}\text { Experiencia de aprendizaje que conecta con la } \\
\text { vida de los estudiantes, ayudándoles a } \\
\text { desarrollar su creatividad y sentido crítico }\end{array}$ \\
\hline $\begin{array}{l}\text { Rodríguez } \\
\text { Cabanillas (2017) }\end{array}$ & $\begin{array}{l}\text { Crear un sendero accesible a } \\
\text { todos los públicos, partiendo } \\
\text { desde la propia localidad }\end{array}$ & $\begin{array}{l}\text { Primaria } \\
\text { CRA }\end{array}$ & $\begin{array}{l}\text { "Red de senderos: una fuente de turismo" } \\
\text { (Proyecto anual: } 24 \text { sesiones) }\end{array}$ & $\begin{array}{l}\text { El Proyecto creado fomenta la participación } \\
\text { activa del alumnado, el trabajo cooperativo y la } \\
\text { resolución de problemas a partir de los saberes } \\
\text { adquiridos }\end{array}$ \\
\hline $\begin{array}{l}\text { Sánchez y López } \\
\text { (2017) }\end{array}$ & $\begin{array}{l}\text { Concienciar, a partir de la salud, } \\
\text { del cuidado del medio ambiente, a } \\
\text { través de la eficiencia energética y } \\
\text { sostenible }\end{array}$ & $\begin{array}{l}5^{\circ} \text { y } 6^{\circ} \text { curso } \\
\text { CRIE }\end{array}$ & $\begin{array}{l}\text { Proyectos: SaludArte y Energía sostenible: } \\
\text { 3.Sol Trabajo interdisciplinar de la salud y } \\
\text { el cuidado del planeta Tierra, durante dos } \\
\text { cursos escolares. }\end{array}$ & $\begin{array}{l}\text { Los proyectos interdisciplinares permiten } \\
\text { aumentar el grado de implicación en el } \\
\text { alumnado, además de adquirir conocimientos } \\
\text { interdisciplinares }\end{array}$ \\
\hline $\begin{array}{l}\text { Ramírez et al. } \\
(2018)\end{array}$ & $\begin{array}{l}\text { Comprobar la incidencia del } \\
\text { programa de intervención sobre la } \\
\text { competencia digital }\end{array}$ & $\begin{array}{l}75 \text { alumnos/as } \\
\left(6^{\circ} \text { curso }\right) \\
\text { CEIP }\end{array}$ & $\begin{array}{l}\text { Proyecto Circus } \\
\text { Proyecto Marbella } \\
\text { Proyecto Olimpiadas }\end{array}$ & $\begin{array}{l}\text { Los resultados confirman que el alumnado } \\
\text { incrementó su competencia digital a nivel } \\
\text { interdisciplinar, con el uso de la metodología } \\
\text { ABP }\end{array}$ \\
\hline
\end{tabular}




\begin{tabular}{|c|c|c|c|c|}
\hline $\begin{array}{l}\text { Rodríguez-Martín } \\
\text { y Buscà (2018) }\end{array}$ & $\begin{array}{l}\text { Aportar una serie de orientaciones } \\
\text { didácticas y competenciales, entre } \\
\text { las áreas de EF y Matemáticas }\end{array}$ & Primaria & Orientaciones didácticas y competenciales & $\begin{array}{l}\text { Este planteamiento favorece tanto la } \\
\text { alfabetización matemática como la } \\
\text { alfabetización motriz }\end{array}$ \\
\hline $\begin{array}{l}\text { Segovia y } \\
\text { Gutiérrez Díaz del } \\
\text { Campo (2018) }\end{array}$ & $\begin{array}{l}\text { Conocer el efecto de una UD de } \\
\text { Educación Deportiva por } \\
\text { Proyectos sobre las competencias } \\
\text { sociales y cívicas, y el sentido de } \\
\text { la iniciativa y espíritu } \\
\text { emprendedor }\end{array}$ & $\begin{array}{l}47 \text { alumnos/as } \\
\left(6^{\circ} \text { curso) }\right. \\
\text { CEIP }\end{array}$ & $\begin{array}{l}\text { "Juegos tradicionales en el mundo" } \\
\text { ( } 21 \text { sesiones) }\end{array}$ & $\begin{array}{l}\text { La Educación Deportiva por Proyectos posee } \\
\text { un gran potencial para mejorar las relaciones y } \\
\text { habilidades sociales, además de mejorar el } \\
\text { grado de autonomía }\end{array}$ \\
\hline $\begin{array}{l}\text { Pérez-Herráez y } \\
\text { Valencia-Peris } \\
\text { (2019) }\end{array}$ & $\begin{array}{l}\text { Utilizar la evaluación formativa y } \\
\text { compartida en un proyecto sobre } \\
\text { los juegos tradicionales }\end{array}$ & $\begin{array}{l}6^{\circ} \text { curso } \\
\text { CEIP }\end{array}$ & $\begin{array}{l}\text { Proyecto interdisciplinar para trabajar los } \\
\text { juegos tradicionales valencianos }\end{array}$ & $\begin{array}{l}\text { Esta propuesta innovadora ha resultado } \\
\text { efectiva, sostenible y replicable en otros } \\
\text { contextos similares }\end{array}$ \\
\hline $\begin{array}{l}\text { Peixoto-Pino et al. } \\
(2019)\end{array}$ & $\begin{array}{l}\text { Dar a conocer un trabajo } \\
\text { interdisciplinar desde el área de } \\
E F \text {, en un centro de Pontevedra }\end{array}$ & $\begin{array}{l}430 \text { estudiantes } \\
\text { de Infantil y } \\
\text { Primaria }\end{array}$ & $\begin{array}{l}\text { Proyecto: "Tú puedes salvar una vida". } \\
\text { curso académico } 2012 / 13 \text {, a través de } 13 \\
\text { actividades globales y actividades } \\
\text { vinculadas a fechas conmemorativas }\end{array}$ & $\begin{array}{l}\text { El contenido sobre la prevención de accidentes } \\
\text { y primeros auxilios es apropiado, ya que mejora } \\
\text { de la convivencia, el cumplimiento de las } \\
\text { normas cívicas y el respeto a los demás }\end{array}$ \\
\hline $\begin{array}{l}\text { Rosa-Guillamón et } \\
\text { al. (2019) }\end{array}$ & $\begin{array}{l}\text { Contribuir al desarrollo de la } \\
\text { competencia motriz y a la } \\
\text { adquisición de las competencias } \\
\text { clave }\end{array}$ & $\begin{array}{l}4^{\circ} \text { curso } \\
\text { CEIP }\end{array}$ & $\begin{array}{l}\text { Proyecto Aventura } \\
\text { (8 sesiones) }\end{array}$ & $\begin{array}{l}\text { Las actividades físicas en el medio natural } \\
\text { desarrolladas a través de proyectos de } \\
\text { aprendizaje permiten el desarrollo integral del } \\
\text { alumnado }\end{array}$ \\
\hline
\end{tabular}




\section{Conclusiones}

Basado en un estudio crítico de los resultados obtenidos queremos centrarnos en algunas cuestiones relevantes encontradas.

El número de publicaciones científicas sobre $\mathrm{ABP}$ en el área de EF en la etapa de Primaria no es muy elevado, aunque es la etapa educativa en donde más estudios se concentran, tal y como comprueban León-Díaz et al. (2018), en una revisión anterior.

En el caso de las investigaciones, al encontrar solamente una, no es posible ofrecer conclusiones fiables y rigurosas, con una base científica consistente. Pero se puede intuir que el estudio, realizado en el 2017, constata que el ABP es una metodología muy motivadora para el alumnado de Primaria, desde el área de EF.

Con respecto a los estudios encontrados sobre propuestas didácticas, se pueden considerar como una innovación en la enseñanza-aprendizaje de la EF actual. Y es que la variedad de contenidos elegidos en estos proyectos (tenis, juegos tradicionales, nuevos deportes, expresión corporal y la adquisición de competencias a través de la $E F)$, hacen que nuestra área sea cada vez más integral y holística. $Y$ anima a que cualquier docente comprometido y con ganas de experimentar nuevas metodologías, pueda aplicarlas.

Por último, los proyectos interdisciplinares encontrados en esta revisión son el grueso de este estudio; lo que nos indica que existen profesionales involucrados con la mejora de la docencia, y concretamente del proceso de enseñanza-aprendizaje de su alumnado. Estos proyectos nacen de las realidades y necesidades educativas encontradas en cada uno de los centros y se enriquecen de su idiosincrasia; ya que además de centros públicos de Infantil y Primaria, los proyectos educativos se han llevado a cabo en centros rurales agrupados y centros rurales de innovación educativa.

Además, el ABP genera un contexto ideal para poder llevarse a cabo en cualquier curso de Primaria, y a través de cualquier contenido del área de EF; siendo una gran ocasión para trabajar contenidos poco habituales del área (Abad et al., 2017; Álvarez e Yllera, 2017; Peixoto-Pino et al., 2019); pudiéndose trabajar interdisciplinarmente desde cualquier área curricular.

Asimismo, el ABP constituye un excelente recurso para la adquisición de las competencias clave en el alumnado; ya que los estudios analizados corroboran que las competencias pueden trabajarse desde esta metodología. Sobre todo, la competencia digital y la competencia social y cívica, a través del trabajo cooperativo y la implicación del alumnado en su proceso de aprendizaje.

Por último y no menos relevante, es necesario que todo proyecto desarrollado desde el área de EF no pierda la esencia del objetivo de la misma en la EP, como es la contribución al desarrollo de la competencia motriz. 


\section{Referencias}

Abad, C., Campo, M.I., Cortés, M.L., y Lienas, F.J. (2017). Cine y expresión corporal: Un proyecto interdisciplinar en una escuela rural. Tándem: Didáctica de la educación física, 56, 26-33.

Álvarez, E., e Yllera, C. (2017). La performance como recurso comunicativo-expresivo en el aula de primaria. El Guiniguada. Revista de investigaciones y experiencias en Ciencias de la Educación, 26, 47-61.

Blázquez, D. (2016). Métodos de enseñanza en educación física. Enfoques innovadores para la enseñanza de competencias. Barcelona: Inde.

Carrera, D. (2016). Como crear nuevos deportes desde la Educación Física. El aprendizaje Por proyectos como estrategia motivante. EmásF. Revista Digital de Educación Física, 38, 103-118. Recuperado de https://dialnet.unirioja.es/servlet/articulo? codigo $=5351995$

Castellar, C., Pradas, F., Rapún, M., Coll, I., y Pérez, S. (2013). Aula en bici: un proyecto longitudinal de intervención docente en Educación Primaria. Retos. Nuevas tendencias en Educación Física, Deporte y Recreación, 23, 5-9.

Contreras, O. R. (2017). La enseñanza por proyectos en Educación Física. En O. Contreras (Ed.), El aprendizaje basado en proyectos en educación física (pp. 9-28). Barcelona: Inde.

De León, S. (2017). Valoración de la metodología de aprendizaje basada en proyectos $(A B P)$ por parte del profesorado de Educación Infantil y Primaria (Trabajo de Fin de Grado de Maestro en Educación Infantil). Universidad de La Laguna, Tenerife.

De Miguel, M. (2009). Metodologías de enseñanza y aprendizaje para el desarrollo de competencias. Madrid: Alianza.

Fernández-Río, J., Calderón, A., Hortigüela, D., Pérez-Pueyo, A., y Aznar, M. (2016). Modelos pedagógicos en educación física: consideraciones teórico-prácticas para docentes. Revista Española de Educación Física y Deportes, 413, 55-75.
García, M., y Baena-Extremera, A. (2017). Motivación en Educación Física a través de diferentes metodologías didácticas. Revista Profesorado, Revista de Currículum y Formación Del Profesorado, 21(1), 387-402. Recuperado de http://www.redalyc.org/pdf/567/56750681 019.pdf

García Carcedo, P. (2018). Escritura creativa e igualdad: versiones de los cuentos tradicionales. Didáctica: lengua $y$ literatura, 30, 87-103.

García-Varcálcel, A. y Basilotta, V. (2017). Aprendizaje basado en proyectos (ABP): evaluación desde la perspectiva de alumnos de Educación Primaria. Revista de Investigación Educativa, 35(1), 113131. doi: 10.6018/rie.35.1.246811

Gil, P. y Sáez, N.M. (2017). Los juegos populares y tradicionales de Don Quijote de la Mancha: Educación Primaria. En O. Contreras (Ed.), El aprendizaje basado en proyectos en educación física (pp. 59-77). Barcelona: Inde.

González, S. Villar, L., Pastor, J.C., y Gil, P. (2013). Propuesta didáctica interdisciplinar en educación primaria en España: la enseñanza de la educación física y el inglés. Paradigma, 34(2), 31-50.

González-Víllora, S. y Prieto-Ayuso, A. (2017). Desarrolla tu propio equipo de Copa Davis: Educación Deportiva Educación Primaria. En O. Contreras (Ed.), El aprendizaje basado en proyectos en educación física (pp. 81-103). Barcelona: Inde.

Hernández, F. (2000). Los proyectos de trabajo: la necesidad de nuevas competencias para nuevas formas de racionalidad. Educar, 26, 39-51.

Hernández, R., Fernández, C., y Baptista, P. (2010). Metodología de la investigación. México: Mcgraw-Hill Interamericana Editores.

Juez, A., y De los Santos, A. (2011). Aprendizaje cooperativo, metodología por proyectos y espacios de fantasía en Educación Física para Primaria. (RE) Construyendo la Expresión Corporal mediante la dramatización de cuentos e historietas 
infantiles. La Peonza: Revista de Educación Física para la paz, 6, 3-23.

Julián, J.A., Ibor, E., Aibar, A., y Aguareles, I. (2017). Educación física, motor de proyectos. Tándem: Didáctica de la educación física, 56, 7-15.

León-Díaz, O., Martínez-Muñoz, L.F. y SantosPastos, M.L. (2018). Análisis de la investigación sobre Aprendizaje basado en Proyectos en Educación Física. Revista Electrónica Interuniversitaria de Formación del Profesorado, 21(2), 27-42.

MEC (2006). Ley Orgánica 2/2006 de Educación. Madrid: MEC.

MEC (2013). Ley Orgánica 8/2013, de 9 de diciembre, para la mejora de la calidad educativa. Madrid: MEC.

Murillo, A., Riaño, M.E., y Berbel, N. (2018). Música con mucho cuento. Eufonía: Didáctica de la música, 76, 7-12.

Núñez, J.L. y León, J. (2015). Autonomy Support in the Classroom. European Psychologist, 20(4), 275-283.

Peixoto, L. (2014). Proyecto interdisciplinar, "escuela: comunidad olímpica". Retos, 25, 140-143.

Peixoto-Pino, L., Rico-Díaz, J. y Arufe, V. (2019). Elaboración y aplicación de un proyecto interdisciplinar en las etapas de Infantil y Primaria sobre prevención de accidentes promovido desde el área de Educación Física. Retos, 35, 250-254.

Pérez-Herráez, I. y Valencia-Peris, A. (2019). Una experiencia de evaluación alternativa en un proyecto interdisciplinar de juegos tradicionales en educación primaria. Infancia, educación y aprendizaje (IEYA), 5(2), 127-131

Ramírez, V., Padial, R., Torres, B., Chinchilla J.L. y Cepero, M. (2018). Consecuencias en la competencia digital del alumnado de primaria de un programa de educación física usando la metodología ABP. Journal of Sport and Health Research, 10(3), 361372.
Rodríguez Cabanillas, J.L. (2017). Red de senderos, una fuente de turismo. Tándem: Didáctica de la educación física, 56, 4245.

Rodríguez-Martín, B., y Buscà, F. (2018). Desarrollar la competencia matemática desde la educación física. Tándem: Didáctica de la educación física, 61, 6672.

Rosa-Guillamón, A., Carrillo-López, P.J. y García-Cantó, E. (2019). Learning based on projects. A didactic experience from the physical education area. ESHPA Education, Sport, Health and Physical Activity, 3(1), 141-159. doi: http://hdl.handle.net/10481/53934

Sánchez, A., y López, M.P. (2017). Salud sostenible: Del cuidado individual al cuidado del planeta. Tándem: Didáctica de la educación física, 56, 16-25.

Segovia, Y., y Gutiérrez Díaz del Campo, D. (2018). Efecto de una unidad didáctica de educación deportiva por proyectos sobre las relaciones sociales $y$ nivel de autonomía. EmásF. Revista Digital de Educación física, 51, 89-103.

Sempere, A., Rovira-Collado, J., Baile, E. (2018). Cómic en el aula de Educación Primaria: propuesta para aprendizajes multidisciplinares. En: Roig-Vila, Rosabel (coord.), Redes de Investigación en Docencia Universitaria (pp.609-617). Alicante: Universidad de Alicante, Instituto de Ciencias de la Educación (ICE).

Trujillo, F. (2014). Aprendizaje basado en proyectos: formación del profesorado de Educación Permanente. En Fernando Trujillo [Blog]. Recuperado de http://fernandotrujillo.es/aprendizajebasado-en-proyectos-formaciondelprofesorado-de-educacionpermanente/

Trujillo, F. (2015). Aprendizaje basado en proyectos. Infantil, Primaria y Secundaria. Madrid: Ministerio de Educación y Ciencia. 